\title{
LOS HOSPITALES MILITARES EN LA ISLA DE CUBA DURANTE LA GUERRA DE 1895-1898
}

\author{
Bonifacio Esteban Marfil
}

Centro de Salud La Chopera, Madrid

\section{RESUMEN}

En este artículo se analiza la creación y desarrollo de la red sanitaria de enfermerías, clínicas y hospitales militares que el Cuerpo de Sanidad Militar puso en funcionamiento durante la guerra de Cuba entre los años 1895 a 1898. La gran cantidad de enfermos originó que muchos de estos centros sanitarios acogieran un mayor número de pacientes que aquel para el que estaban inicialmente diseñados, y que se instalaran en edificios y locales que muchas veces no reunían las condiciones técnicas e higiénico-sanitarias adecuadas.

PALABRAS CLAVE: sanidad militar, guerra de Cuba, asistencia médica, siglo XIX.

\section{SUMMARY}

In this article I analyse the development of the sanitary network of infirmaries, clinics and military hospitals that the Military Medical Corp achieved during the war of Cuba from 1895 to 1898. The great amount of sick people made many sanitary centres receive more patients than they were designed for, also they were established in places that, many times, did not have adequate technical or sanitary conditions.

KEY WORDS: military health, Cuba's war, medical care, $19^{\text {th }}$ century.

La guerra de Cuba entre los años 1895 a 1898 supuso para el ejército español un desafío importante. Fue necesario movilizar grandes contingentes de hombres $^{1}$ que eran trasladados al otro lado del Atlántico y se hizo frente a una

1 Fueron enviados a la Isla de Cuba aproximadamente unos 220.000 hombres entre los años 1895-1898. Ver: YÁNEZ GALLARDO, C. R. (1992), «La última invasión armada. Los contingentes militares españoles a las guerras de Cuba, siglo XIX», Revista de Indias, 52 (194), 107-126, p. 110. 
Plano de la Habana con sus establecimientos sanitarios.

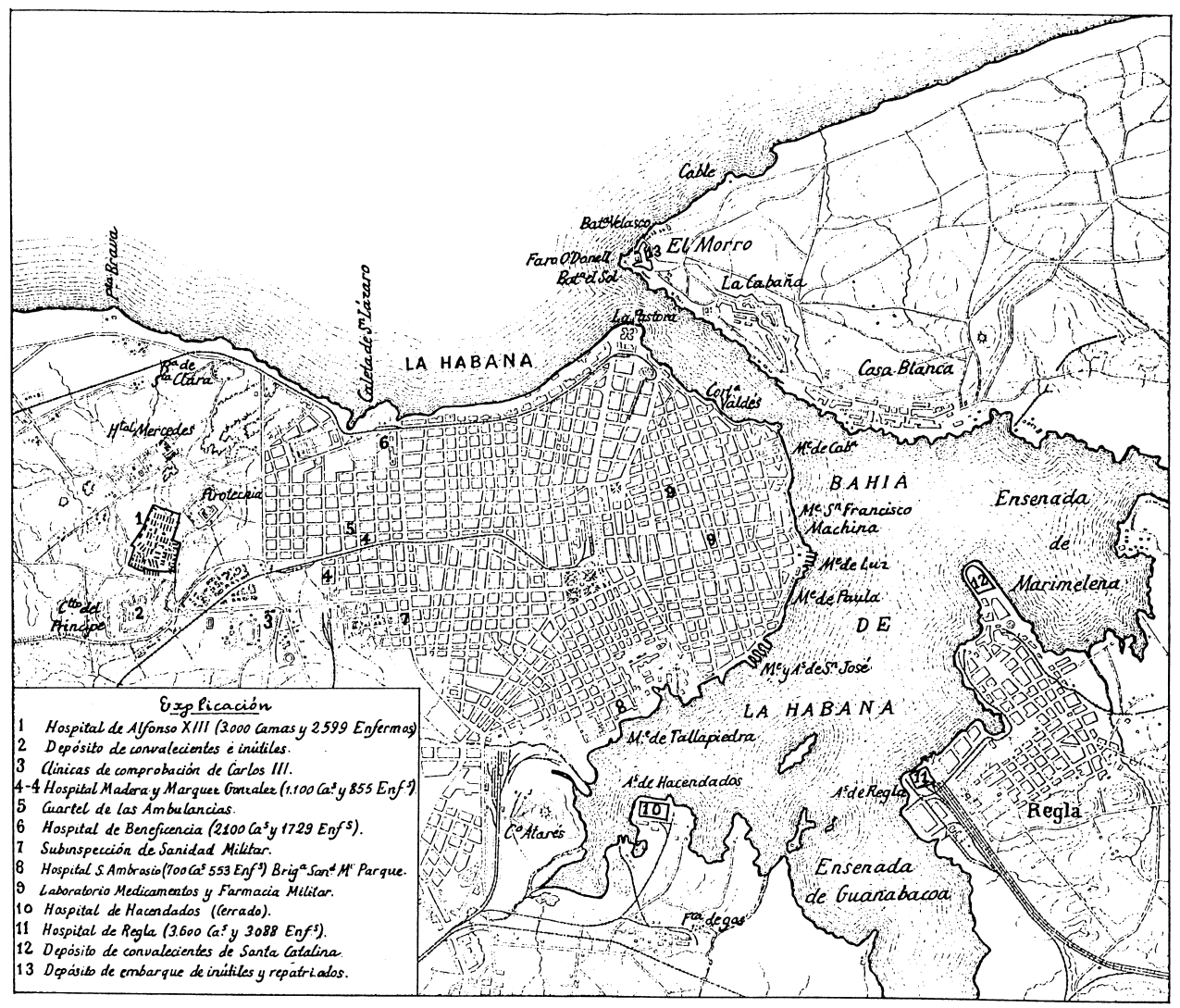

Escala aproximada 1:36.000.

dura campaña, combatiendo contra un enemigo - los mambises - en continuo movimiento; que utilizaban tácticas de guerrilla y que vivían sobre el terreno en el campo y en la manigua. Pero, como ya se ha perfilado en otros estudios, el principal enemigo no fue tan sólo el plomo o el machete cubano, sino también las enfermedades, tales como la fiebre amarilla o el paludismo,

También formaron parte del Ejército Español un alto número de voluntarios, cubanos u oriundos de España afincados en Cuba; y aunque no se conoce su cifra exacta parece ser que podrían haber sido unos 50.000 ó 60.000 hombres a lo largo de toda la campaña. Ver: Anuario Militar de España, Año 1896, Madrid, pp. 754-755. Anuario Militar de España, Año 1897, Madrid, pp. 750, 782-783. Anuario Militar de España, Año 1898, Madrid, pp. 816-817. 


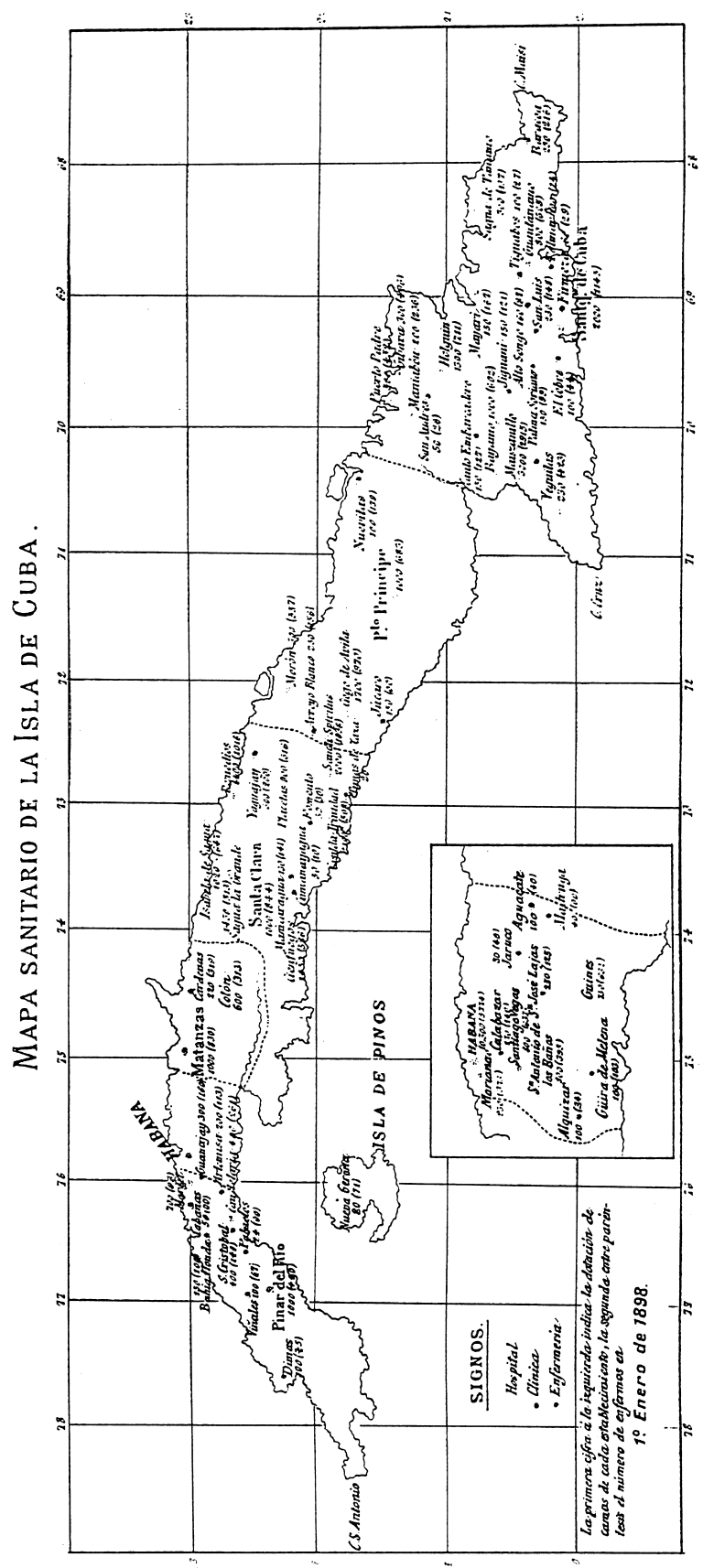


que diezmaron rápidamente las filas españolas ${ }^{2}$. El ejército español se vio inmerso de esta manera en una guerra calificada de «auténtica campaña sanitaria», donde uno de los mayores retos fue el derivado de la masiva asistencia sanitaria que hubo de hacerse a las tropas ${ }^{3}$. En este estudio se realiza un recorrido por uno de los aspectos de esta atención sanitaria al analizar la puesta en marcha y desarrollo de la red de asistencia que a través de enfermerías, clínicas y hospitales se realizó durante aquella campaña militar.

El Cuerpo de Sanidad Militar, antes de iniciarse la guerra en 1895, contaba en Cuba con una infraestructura muy elemental de hospitales y enfermerías militares, ya que habían de atender a un pequeño contingente de tropas en la isla. Los establecimientos existentes eran los siguientes: hospital militar de $\mathrm{La}$ Habana, Santiago de Cuba, Santa Clara y Puerto Príncipe, y algunas enfermerías en poblaciones que tuvieran una comunicación dificultosa con estos hospitales $^{4}$. Durante la guerra de Cuba, entre los años 1895 y 1898, debido al importante incremento en el número de enfermos, hubieron de ampliarse los existentes e inaugurarse nuevas enfermerías y hospitales, que sufrieron asi-

2 Entre la bibliografía existente sobre la guerra de Cuba existen algunos estudios sobre los aspectos sanitarios que fueron inherentes a la misma. Para dar una idea de la situación se pueden consultar entre otras: DíAZ MARTíNEZ, Y. (1998), «La sanidad militar del Ejército Español en la Guerra de Cuba en 1895», Asclepio, Vol.-L-1, 159-173. Esteban MARFIL, B. (2000), La Sanidad Militar Española en la Guerra de Cuba (1895-1898), UAM, tesis doctoral inédita. PASCUAL MARTínEZ, P. (1996), «Combatientes, muertos y prófugos del Ejército Español en la guerra de independencia de Cuba (1895-1898)», Estudios de Historia Social y Económica, 13, 479-485. HERNÁNDEZ SANDOICA, E.; MANCEBO, M. F. (1978), «Higiene y sociedad en la guerra de Cuba (1895-1898). Notas sobre soldados y proletrios, Estudios de Historia Social,5, 361-384.

3 Durante el año 1895 fueron asistidos 49.485 hombres, durante 1896 el número de asistencias hospitalarias fue de 238.235, para ascender a la desmesurada cifra de 625.165 ingresos durante 1897. (Los efectivos militares en estos años podrían cifrarse en 100.000 hombres para el primer año y 200.000 hombres para los otros dos respectivamente). Obviamente estas cifras nos indican que muchos soldados sufrían varios reingresos en el mismo año. Tampoco aparecen aquí contabilizados los soldados que eran atendidos y tratados en las columnas de operaciones, en ingenios o en pequeños poblados aislados. Datos extraídos de:

LARRA y CEREZO, A. (1898), Les hôpitaux militaires de l'T̂le de Cuba et notamment l'hôpital d'Alphonse XIII de La Havane pedant la guerre actuelle, Impremerie du Dépot de la Guerre, Madrid, pp. 9,12,13.

LARRA y CEREZO, A. (1901), Datos para la historia de la campaña sanitaria en la guerra de Cuba (apuntes estadísticos relativos al año 1896), Madrid, Imprenta de Ricardo Rojas, pp. 19, 26-27.

Memoria Resumen de la Estadística Sanitaria del Ejército Español, Año 1897, Madrid, Imprenta y Litografía del Depósito de la Guerra, (1899), p. 43.

4 LARra y CEREZO, A. (1898), p. 7. 
mismo continuas ampliaciones. Todo esto se realizó siempre según los avatares y necesidades de la campaña y en relación directa con el desarrollo de las operaciones militares. Esto originó que hospitales provisionales terminaran siendo permanentes, que pequeñas enfermerías acabaran convertidas en auténticas clínicas, o que se cerrasen determinados establecimientos para ser a veces reabiertos más adelante.

Los establecimientos sanitarios presentes en la Isla de Cuba se pueden dividir en:

- Enfermerías regimentarias, que daban asistencia a un regimiento o unidad militar concreta, dependiendo a todos los efectos del batallón o regimiento para el que eran creadas. Durante las operaciones las enfermerías tuvieron el carácter de hospital móvil o semimóvil de campaña y también de evacuación a los hospitales más fijos.

- Clínicas, que eran centros con personal y administración propios pero dependientes de otro hospital fijo de mayor importancia, como si se tratase de una ampliación del mismo. Eran en realidad hospitales pequeños capaces de incrementar su número de camas según las necesidades del momento y variando incluso su punto de residencia si la movilidad de las operaciones lo hacía preciso.

- Hospitales fijos, que radicaban habitualmente en poblaciones de importancia y se dividieron en permanentes y provisionales que eran los que se crearon por necesidades de la campaña, que fueron la mayoría.

RÉGIMEN INTERNO Y DISTRIBUCIÓN EN LOS ESTABLECIMIENTOS SANITARIOS MILITARES.

Los establecimientos sanitarios antes de su apertura eran inspeccionados por el Cuerpo de Ingenieros que realizaban un detallado informe sobre los locales donde se iban a establecer, indicando las reformas y mejoras que fueran pertinentes para adecuarlos a su nueva función. Sin embargo, éstos tenían que ser rápidamente ampliados o debían recibir mayor número de enfermos que los inicialmente considerados. En todos los establecimientos sanitarios había una separación entre las camas destinadas para los enfermos y heridos de la tropa y unas pocas reservadas para los oficiales ${ }^{5}$. La distribución de los

5 Archivo Histórico Militar, Madrid, Sección Ultramar, Fondo: Capitanía General de Cuba, Caja 1216, 1 legajo. 
hospitales era en salas, existiendo una separación entre salas de enfermos de medicina y de cirugía.

Dentro de los enfermos de medicina formaban un capítulo aparte los afectados por enfermedades infecto-contagiosas, como el paludismo y la fiebre amarilla. Especialmente la segunda, ya que debido a la creencia de su alta transmisibilidad por contacto los enfermos se situaban siempre en salas aisladas o mejor aún en pabellones convenientemente separados del resto de las instalaciones sanitarias ${ }^{6}$. En los hospitales y enfermerías militares de la isla eran atendidos todos los individuos componentes del ejército español, ya fuesen de los batallones expedicionarios o perteneciesen a los cuerpos de voluntarios cubanos. Siendo estos últimos igualmente incluidos en las estadísticas de morbilidad y mortalidad de los nosocomios

\section{PERSONAL SANITARIO FACULTATIVO ${ }^{7}$.}

El personal sanitario facultativo de los hospitales y enfermerías militares estaba constituido por los médicos y farmacéuticos. Éstos podían ser médicos o farmacéuticos militares profesionales o provisionales de Sanidad Militar, existiendo también médicos y farmacéuticos auxiliares, contratados en Cuba, para ayudar en el servicio de los establecimientos sanitarios. La plantilla básica de los hospitales de la Isla estaba compuesta por un director médico, un jefe de servicios, los jefes de clínica y un jefe de farmacia; además de un auditor y un administrador. El número de médicos de plantilla de los distintos hospitales y enfermerías solía estar en relación directa con el número de camas del nosocomio ${ }^{8}$.

6 Instrucciones higiénicas para el Ejército de la Isla de Cuba, (1896), La Habana, Imprenta de A. Álvarez y comp., p. 22-25, 57-58.

7 Para ampliar la información relativa a los médicos que atendieron a las tropas españolas en Cuba puede consultarse: ESTEBAN MARFIL, B. (2002), «El esfuerzo sanitario de un ejército colonial en guerra, (1895-1898)», Las ciudades y la guerra, 1750-1898, BROSETA et al. (eds.), pp. 489-507.

8 Un hospital como el de Ciego de Ávila, de 500 camas, disponía en noviembre de 1895 de un director médico (médico mayor), un jefe de servicios (médico mayor), cuatro jefes de clínica (médicos primeros), un jefe de farmacia, y un administrador. En Diciembre de 1897, contando ya con 1.700 camas, su plantilla se había aumentado con otros dos médicos segundos y tres médicos militares provisionales. El hospital militar de Sancti Spíritus, dotado inicialmente con 150 camas, estaba atendido en Noviembre de 1895 por: tres médicos mayores, tres médicos primeros, un médico segundo y dos farmacéuticos. En Diciembre de 1897, 
La idea que subyace al comparar en los Anuarios Militares el personal destinado en los hospitales y enfermerías es la de que este personal fue aumentando progresivamente a medida que eran ampliadas estas clínicas. Aunque la plantilla médica no se incrementaba de una forma directamente proporcional al gran aumento de nuevas camas con que iban siendo dotados estos establecimientos, por lo que los médicos hubieron de atender progresivamente a mayor número de pacientes. Debido precisamente a esta escasez relativa de médicos militares suficientes para atender a toda la población hospitalaria, fue por lo que se hizo preciso contratar los servicios de médicos civiles cubanos en calidad de médicos auxiliares de sanidad militar9.

\section{LA RED SANITARIA DE CUBA.}

A continuación realizaremos un examen, desde Occidente a Oriente, por las distintas provincias de la isla de Cuba: Pinar del Río, La Habana, Matanzas, Santa Clara (Las Villas), Puerto Príncipe (Camagüey) y Santiago de Cuba, detallando los centros sanitarios con que contó cada zona, ordenados cronológicamente según su fecha de inauguración, indicando su categoría y el número de camas de que disponían. También se analizarán, en algunas ocasiones, determinados hospitales, su personal, su funcionamiento y sus problemas ${ }^{10}$.

habiendo alcanzado las 1.500 camas, su plantilla estaba compuesta por un subinspector médico de $2^{\mathrm{a}}$ clase, cuatro médicos mayores, cuatro médicos primeros, un médico segundo, dos médicos auxiliares y dos farmacéuticos. Se puede consultar la plantilla médica de los hospitales y enfermerías militares en los Anuarios Militares publicados en estos años. Ver: Anuario Militar de España, año 1896, Madrid, Imprenta y litografía del Depósito de la Guerra, 1896, pp. 733-755. Anuario Militar de España, año 1898, Madrid, Imprenta y litografía del Depósito de la Guerra, 1898, pp. 783-817.

9 Sus contratos eran realizados sólo por el tiempo que fuese precisa su colaboración (lo cual variaba según las circunstancias de la campaña y la época del año), cobrando 50 pesos mensuales por el servicio de visita o 90 pesos mensuales si realizaban visita y guardias. Casi todos los hospitales de la Isla requirieron en algún momento de ellos, así los podemos encontrar en los hospitales y enfermerías de poblaciones como: La Habana, Marianao, Sagua la Grande, Pinar del Río, Matanzas, Candelaria, Cárdenas, Santiago de Cuba, Sancti Spíritus, Remedios, Nuevitas, Puerto Padre, Holguín, Santa Clara, Trinidad, Güines, Isabela, Guantánamo, Yaguajay, Manzanillo, etc.

10 Estos listados de las instalaciones sanitarias presentes en las distintas zonas de la Isla de Cuba, que pretenden ser exhaustivos, han sido elaborados a partir de las siguientes fuentes: LARRA y CEREZO, A. (1898), Appendice I: Carte sanitaire de l'Île de Cuba. LARRA y CEREZO, A. (1901), pp. 22-25. WEYLER, V. (1910), Mi mando en Cuba, Madrid, Imprenta Litográfica y Casa 


\section{Provincia de Pinar del Río}

Hasta esta provincia, la más occidental de la isla, llegó la «columna de invasión» de los «insurrectos» cubanos, acaudillados por Antonio Maceo ${ }^{11}$. De esta manera se trasladó el conflicto armado hasta las provincias occidentales de la Isla, que no habían sufrido los efectos de la guerra de $1868^{12}$. Tras hacerse cargo del mando, el general Weyler decide comenzar las operaciones militares más importantes en la provincia de Pinar del Río; trasladándose gran cantidad de soldados a esta zona para guarnecer los poblados, construir y defender la trocha de Mariel a Majana y operar en columnas ${ }^{13}$.

La consecuencia directa del acúmulo de tropas en condiciones poco higiénicas y de los trabajos en la trocha, muchas veces en zonas pantanosas e insalubres, fue el incremento importante de las enfermedades infecto-contagiosas: paludismo, disentería, fiebre amarilla, etc. No existiendo, previamente a la guerra, hospitales militares en esta zona de la Isla fue necesaria la creación urgente de diversos hospitales, clínicas y enfermerías militares, que pronto se vieron repletas de enfermos, sobre todo en los meses de Agosto a Octubre de

Editorial de Felipe González Rojas, vol. 5 , pp. 125-126. Archivo Histórico Militar Madrid, Sección Ultramar, Fondo: Capitanía General de Cuba, Caja 214, Archivo General Militar, Negociado de Sanidad de Cuba. SENAE y VicENTE, C. (1898), Estudio crítico de los servicios generales de los Hospitales de la Isla de Cuba con motivo de la campaña actual, por el Médico Mayor del Cuerpo de Sanidad Militar, D. Clemente Senae y Vicente, La Habana, manuscrito inédito.

11 La «invasión de Occidente» culmina cuando las tropas de Maceo entran en Mantua, la población más occidental de la Isla, el 22 de Enero de 1896. Esto significó además el fracaso de la política militar desarrollada por el General Martínez Campos, que presentó su dimisión, siendo relevado de su cargo el 16 de Enero de 1896. Ver: PAYNE, Stanley G.(1977), Ejército y sociedad en la España liberal. 1808, 1936., Madrid, Akal, pp. 113-114.

12 La primera guerra hispano-cubana, entre los años 1868-1878, circunscribió su campo de acción tan sólo a las provincias orientales de la Isla.

13 El general Valeriano Weyler arribó a la Habana el 10 de Febrero de 1896, encontrando el país en una situación comprometida. Puso rápidamente en marcha un plan para vencer sucesivamente la insurrección desde Occidente hacia Oriente. Para ello en una primera etapa, que abarcó desde el 10 de Febrero al 20 de Marzo de 1896, dedicó grandes contingentes de tropas españolas a la construcción de una línea defensiva y de observación entre las poblaciones de Mariel y Majana, con el propósito de arrinconar a Maceo en la provincia de Pinar del Río. Y en una segunda etapa, desde el 20 de Marzo al 10 de Octubre de 1896, el Ejército Español se consagró a la definitiva separación de los efectivos de Antonio Maceo en Pinar del Río de los de Máximo Gómez en Camagüey, a la vez que se perseguía y hostigaba activamente a los insurrectos en Pinar del Río. Ver: AlONSO BAQUeR, M. (1997), «Las campañas en Cuba del general Weyler», en Ramos, D.; Diego, E. (coord.), Cuba, Puerto Rico y Filipinas en la perspectiva del 98, Madrid, Editorial Complutense, pp. 25-26. 
1896, cuando las enfermedades infecciosas tenían mayor prevalencia, lo cual obligó a evacuar a muchos de estos enfermos a los hospitales de La Habana ${ }^{14}$.

\section{HOSPITALES, CLÍNICAS Y ENFERMERÍAS MILITARES DE LA PROVINCIA DE PINAR DEL RÍO:}

\begin{tabular}{|l|c|l|c|c|}
\hline NomBRE & FECHA CREACIÓN & ESTABLECIMIENTO & CAMAS & $\begin{array}{c}\text { CAMAS } \\
\text { INICIALES }\end{array}$ \\
FINALES
\end{tabular}

14 La existencia de un ferrocarril central que cruzaba la provincia desde la población de Pinar del Río hasta La Habana, fue determinante en la evacuación de los enfermos y heridos. Otras veces se utilizaban también líneas costeras de vapores de cabotaje para esta función.

15 Entre corchetes aparecerá en este cuadro y los siguientes el nombre del hospital fijo del que dependía cada clínica.

16 En Artemisa existía además de la clínica un depósito de transeúntes. Los depósitos de transeúntes y enfermos existían en muchas poblaciones de la Isla; estaban atendidos por personal no sanitario y su principal misión era acoger a los enfermos leves que iban a los hospitales o a los convalecientes que regresaban de los mismos. Éste de Artemisa estaba situado en un barracón a las afueras del poblado y cerca de la estación de ferrocarril. Acogía a los soldados destinados o procedentes de los hospitales de La Habana o Santiago de las Vegas. Aquí se les facilitaba auxilio, alimento y cama a los soldados mientras esperaban ser remitidos a los hospitales o a sus respectivas unidades; y si alguno precisaba atención médica era trasladado al hospital de la población. Vicente Rodríguez Vargas en sus memorias refleja bien la actividad en estos establecimientos, puesto que él estuvo destinado en el Depósito de transeúntes de Artemisa desde el 9 de Octubre de 1896 hasta el 1 de Agosto de 1897. RodrígueZ VARGAS,V. (1995), Un año en Cuba, 1897. Notas de mi diario de campaña, Gráficas SUS-SE, Valladolid, (Edición familiar). 
Durante el año 1896 se produjeron 27.483 ingresos en los hospitales y enfermerías militares de la provincia de Pinar del Río; al finalizar el año permanecían ingresados 2067 enfermos. Los que mayor número de pacientes ingresados registraron a lo largo del año fueron los de Guanajay, Pinar del Río, Bahía Honda y Mariel ${ }^{17}$. Concretamente el hospital de Guanajay ${ }^{18}$ llegó a recibir unos 14.000 enfermos durante los meses críticos de la campaña en esta zona de la Isla, de los cuales unos 7.000 fueron evacuados hacia otros hospitales, principalmente de La Habana ${ }^{19}$. A fecha del $1^{\circ}$ de Enero de 1898 el número de camas disponible en los establecimientos sanitarios de esta provincia sumaba 3.424; habiendo sólo ingresados 1.427 individuos en ese momento ${ }^{20}$.

\section{Provincia de La Habana}

Antes del inicio de la guerra existía en la capital de la isla, La Habana, tan sólo el Hospital General Militar. Ya se ha comentado como la propagación de la guerra hasta las provincias más occidentales de la Isla y el consecutivo plan de campaña del general Weyler, se tradujo en un importante incremento del número de enfermos en las regiones de Pinar de Río, La Habana y Matanzas. De manera que gran parte de los enfermos que no pudieron ser absorbidos por los hospitales de Pinar del Río hubieron de enviarse a los hospitales de La Habana. Esto motivó la apertura de nuevos hospitales militares en la provincia y capital de La Habana, los cuales aparecen recogidos en el siguiente listado.

Durante el año 1896 ingresaron en los hospitales de La Habana, capital y provincia, 95.611 enfermos; permaneciendo ingresados 13.359 al finalizar el año. De estos ingresos 75.735 lo fueron en los hospitales de La Habana capital y los restantes en los otros hospitales y enfermerías de la provincia, especialmente el de Santiago de las Vegas que registró 10.437 ingresos en este año ${ }^{21}$. En Enero de 1898 el número de camas disponible en la provincia de La Habana eran 11.590, habiendo ingresados 11.443 pacientes en ese momento ${ }^{22}$.

17 Larra y Cerezo, A.(1901), p. 17.

18 El Hospital de Guanajay se encontraba en dicha población, estratégicamente situado en el límite entre las provincias de Pinar del Río y de La Habana, se encontraba además en comunicación directa por ferrocarril con Artemisa, importante poblado situado en el centro de la trocha de Mariel a Majana.

19 Senae y Vicente, C.(1898), p. 28.

20 Larra y Cerezo, A. (1898), Appendice I: Carte Sanitaire de l'Île de Cuba.

21 LARRA y CEREZO, A. (1901), p. 17.

22 LARra y CEREzo, A. (1898), Appendice I: Carte sanitaire de l'Île de Cuba. 


\section{HOSPITALES, CLÍNICAS Y ENFERMERÍAS MILITARES DE LA PROVINCIA Y CAPITAL DE LA HABANA:}

\begin{tabular}{|l|l|l|c|c|}
\hline NoMBRE & FECHA CREACIÓN & ESTABLECIMIENTO & $\begin{array}{c}\text { CAMAS } \\
\text { INICIALES }\end{array}$ & $\begin{array}{c}\text { CAMAS } \\
\text { FINALES }\end{array}$ \\
\hline $\begin{array}{l}\text { Santiago de las } \\
\text { Vegas }\end{array}$ & 29 Junio 1895 & Hospital & 200 & 800 \\
\hline Alfonso XIII & 23 Enero 1896 & Hospital & 2.000 & 3.000 \\
\hline Calabazas & 13 Mayo 1896 & $\begin{array}{l}\text { Clínica (Santiago de las } \\
\text { Vegas) }\end{array}$ & 100 & 170 \\
\hline $\begin{array}{l}\text { San Antonio de } \\
\text { Baños }\end{array}$ & 13 Junio 1896 & Hospital & 510 & 800 \\
\hline Marianao & 13 Junio 1896 & Clínica $\rightarrow$ Hospital & 200 & 950 \\
\hline Ingenio Toledo & 16 Junio 1896 & Clínica (Alfonso XIII) $\rightarrow$ Depósito convalecientes \\
\hline Aguacate & 30 Julio 1896 & Enfermería & 50 & 100 \\
\hline Madera & 9 Agosto 1896 & Hospital & 500 & 1.100 \\
\hline $\begin{array}{l}\text { San José de las } \\
\text { Lajas }\end{array}$ & 9 Agosto 1896 & Clínica (Alfonso XIII) & 150 & \\
\hline Güines ${ }^{23}$ & 23 Agosto 1896 & Hospital & 200 & 750 \\
\hline Beneficencia & 28 Agosto 1896 & Hospital & 800 & 2100 \\
\hline Regla & 11 Octubre 1896 & Hospital & 500 & 3600 \\
\hline San Ambrosio & 21 Octubre 1896 & Hospital & 700 & \\
\hline $\begin{array}{l}\text { Marqués Gonzá- } \\
\text { lez }\end{array}$ & 28 Octubre 1896 & Clínica (Madera) $\rightarrow$ Hospital & 500 & \\
\hline Santa Catalina & 12 Noviembre 1896 & Clínica (Regla) $\rightarrow$ Hospital & 500 & \\
\hline Hacendados & 13 Noviembre 1896 & Hospital & 1000 & \\
\hline Alquízar & 12Diciembre 1896 & Enfermería & 100 & \\
\hline Güira de Melena & 19 Diciembre 1896 & Enfermería & 50 & \\
\hline Jaruco & 1 Septiembre 1897 & Clínica (Alfonso XIII) & & \\
\hline Palos & 2 Diciembre 1897 & Enfermería & & \\
\hline
\end{tabular}

23 El hospital militar de Güines estaba instalado en el edificio que ocupaba el hospital civil que fue cedido por el ayuntamiento. Al poco tiempo de iniciar su andadura muchos de estos hospitales se veían saturados por el alto número de ingresos; así en éste de Güines había alojados 268 enfermos en Octubre de 1896, por lo que se vio obligado a evacuar periódicamente a parte de sus enfermos menos graves a los hospitales de la capital, a fin de poder seguir recibiendo a otros más graves. Archivo Histórico Militar, Sección Ultramar, Fondo: Gobiernos Militares, Caja 202, 5 legajos. 
El alto número de ingresos que se produjo en los hospitales y enfermerías de La Habana se debió, inicialmente, al hecho de tener que absorber parte de los enfermos provenientes de Pinar del Río; aunque fueron otras dos las causas principales, la primera es que en esta ciudad estaban los mejores hospitales de la Isla, por lo que eran punto obligado de referencia al que se trasladaban muchos enfermos, y la segunda era que en La Habana se realizaban los exámenes de los enfermos que eran candidatos a ser repatriados, llegando por este motivo a la capital multitud de éstos provenientes de toda la Isla ${ }^{24}$. Los Hospitales situados en la ciudad de La Habana fueron: Alfonso XIII, Beneficencia, Hacendados, Madera, Marqués González, Regla, Santa Catalina y San Ambrosio.

\section{La ciudad de La Habana. Su situación higiénico-sanitaria}

La Habana era una ciudad moderna y cosmopolita en aquella época, punto de llegada de muchas de las expediciones militares que provenían de la Península, punto de partida de los repatriados, sede del poder político y militar de la metrópoli y también una ciudad con importantes problemas sanitarios. El médico cubano Acosta en una memoria presentada, poco antes de iniciarse la guerra, al congreso Panamericano celebrado en Washington nos da una imagen de la deficiente situación sanitaria de la ciudad. Comenta el autor que los establecimientos sanitarios de la capital eran verdaderos focos de infección, sobre todo de la fiebre amarilla, no existiendo en algunos salas aisladas para esta enfermedad. No había tampoco inodoros y se empleaba el sistema de bacinillas, al igual que en el hospital militar, que se limpiaban dos veces al día ${ }^{25}$. Situación que también se puede contrastar en el cuestionario publicado por Cesáreo Fernández Losada, jefe de sanidad militar en la isla de Cuba durante gran parte de la guerra, al poco tiempo de hacerse cargo de su puesto publicó en 1896: Cuestionario sobre el saneamiento de la Habana ${ }^{26}$.

24 El procedimiento que seguían los enfermos para pasar estos reconocimientos aparece reflejado en: RoDRÍGUEZ VARGAS, V. (1995), pp. 39-42, 44-46.

25 Estos aspectos de la ciudad vienen extractados de la «Memoria que el Dr. Acosta presentó al Congreso Panamericano de Washington en el artículo «El Hospital Militar de la Habana», Revista de Sanidad Militar, 9 (181), 1895, pp. 14-16.

26 La elaboración y publicación de este documento por el máximo responsable de la Sanidad Militar española en la Isla, en el que se analizan los principales problemas de salubridad de La Habana y se proponen soluciones a éstos, de acuerdo a los principios higiénico-sanitarios más avanzados de la época, nos pone de manifiesto el interés del Cuerpo de Sanidad militar por abordar 


\section{El Hospital General Militar de La Habana}

Este hospital, también denominado del Príncipe, se encontraba situado en la que fue la antigua factoría de tabacos de La Habana; a donde se había trasladado el antiguo hospital militar de San Ambrosio, por necesidad de ampliación del mismo en el año 1842, por lo que en muchas partes se le seguía denominando con su antiguo nombre ${ }^{27}$. En palabras del inspector de sanidad militar Pedro Peñuelas, existía una «carencia absoluta de todas las condiciones necesarias del edificio para el objeto a que se le ha destinado ${ }^{28}$. En las salas de cirugía era imposible la correcta asepsia por las malas condiciones de las mismas, faltando también los medios necesarios para la completa desinfección de los instrumentos de cirugía. Las salas de medicina eran en su mayoría «un verdadero ataque a la higiene hospitalaria $»^{29}$. La sala de infecciosos no se encontraba suficientemente aislada. El hospital tampoco contaba con alcantarillado, por lo que las deposiciones y orina de los enfermos habían de permanecer en las salas hasta la hora de la limpieza, en que eran sacados los orinales de mano y sillicos para verter su contenido en depósitos que eran llevados al muelle para verterlo en: «el gran depósito de inmundicias, la bahía, que es por sí misma una causa más del mefitismo que impera en el hospital ${ }^{30}$. Por todas estas razones estimaba Peñuelas que era imprescindible la edificación de otro hospital militar que reuniera las condiciones que demandaba la ciencia moderna.

El hospital militar fue clausurado a finales de 1895, cuando se inaugura el Hospital del Príncipe (más tarde denominado Hospital Militar Alfonso XIII). Pero el importantísimo número de enfermos que se originó en la guerra obligó a su reapertura el 21 de octubre de 1896, dotándolo inicialmente con 700 camas y denominándolo Hospital de San Ambrosio.

los problemas sanitarios de la Isla de Cuba de una forma integral. Véase: FERNÁNDEZ LOSADA, C. (1896), Cuestionario sobre el saneamiento de La Habana, La Habana, Guerra Hnos. y Compa.

27 Guerra, F. (1994), El hospital en Hispanoamérica y Filipinas 1492-1898, Madrid, Ministerio de Sanidad y Consumo, pp. 111-112, 129-130.

28 Estas ideas están recogidas en una memoria que presentó Pedro Peñuelas al Excmo. Sr. Capitán General de Cuba el 27 de Julio de 1894, y que aparece publicada como: «El Hospital Militar de La Habana» Revista de Sanidad Militar, 9 (181), 1895, pp. 11-16.

29 Ibidem, p. 13.

30 Ibidem, p. 15. 


\section{Hospital Militar Alfonso XIII.}

Este hospital, fruto de los desvelos anteriormente comentados y de las necesidades sanitarias acuciantes que esta nueva campaña de 1895 supuso, fue inaugurado a finales de 1895 bajo el mandato del general Arsenio Martínez Campos y siendo jefe de la sanidad militar de la isla Pedro Peñuelas ${ }^{31}$, con el nombre de Hospital del Príncipe, puesto que venía a sustituir al anteriormente reseñado. Aunque fue concebido para unas 500 camas, fue pronto ampliado hasta las 2.000, alcanzando finalmente unas 3.000 camas, cuando era jefe de sanidad militar de la isla Cesáreo Fernández Losada. Rebautizado con el nombre de Alfonso XIII, comenzó su nueva andadura el 23 de enero de 1896.

El hospital estaba ubicado sobre una colina a dos kilómetros de La Habana. Se construyó siguiendo el sistema de pabellones aislados de madera, enlazados por galerías cubiertas; y fue considerado uno de los mejores en su tiempo, tanto por sus instalaciones y servicios como por la calidad y cantidad de atención sanitaria que prestó durante la guerra de Cuba. En cuanto a la plantilla del hospital, el primer director fue el subinspector médico de $1^{\text {a }}$ clase Juan Merino Aquinaga. En el año 1896 había destinados 26 médicos del cuerpo, 3 médicos auxiliares y 2 farmacéuticos y de personal subalterno: 150 individuos; durante el año 1897 hubo aquí destinados por término medio unos 23 médicos del cuerpo de sanidad militar, médicos mayores, primeros y provisionales, 4 médicos auxiliares, y un subinspector de farmacia ayudado por un farmacéutico auxiliar civil, y 170 miembros de la brigada sanitaria. La asistencia sanitaria que se prestó en el hospital durante estos años fue muy importante, habiendo recibido más de 80.000 enfermos en el transcurso de dos años $(96,97)^{32}$.

31 Pedro Peñuelas y Fornesa fue inspector médico de $2^{\mathrm{a}}$ clase desde 1892. Habiendo sido destinado a Cuba, ejercía el puesto primer jefe del Cuerpo de Sanidad Militar cuando estalló la insurrección. Falleció en octubre de 1895 en La Habana a consecuencia de una fiebre palúdica perniciosa.

32 Ángel de Larra y Cerezo, que fue jefe de clínica en este hospital, nos describe sus instalaciones a finales de 1897 de la siguiente manera. Disponía de cuatro salas para oficiales, una de ellas al menos para heridos y otra para los atacados de fiebre amarilla. Los enfermos de medicina y cirugía, incluidos los heridos y las especialidades fueron destinados a 50 clínicas, que estaban situadas en pabellones separados entre sí. Los departamentos de infecciosos, convenientemente aislados, estaban compuestos por 12 barracones con cocina y una pabellón donde vivían los médicos de guardia. Una sala de operaciones, perfectamente aséptica y bien provista de material quirúrgico, que era constantemente renovado. La farmacia, ocupando una gran pabellón, formando el departamento sucursal de medicamentos, siendo uno de los dos existentes en la La Habana. La farmacia se surtía del Laboratorio Central de Madrid y de la 


\section{Hospital militar de Regla.}

En la ensenada de Guanabacoa, frente a La Habana, y junto a una estación de ferrocarriles, estaban situados los almacenes de Regla. En estos antiguos almacenes de azúcar, al inicio de la guerra, estaban siendo alojados los soldados de distintas expediciones procedentes de la Península ${ }^{33}$. La necesidad de espacios físicos adecuados para acomodar a la ingente cantidad de enfermos que había, obligó a las autoridades militares a ponerse en contacto con la Compañía de Ferrocarriles Unidos y Almacenes de Regla, dueña de dichos inmuebles, con el fin de abrir en sus instalaciones un hospital militar provisional.

Comenzó a funcionar como tal el 11 de Octubre de 1896 con 600 camas instaladas en el almacén $n^{\circ} 6$; encargándose el Parque Sanitario y el Laboratorio de La Habana de suministrar urgentemente los instrumentos, material sanitario, medicinas y utensilios de farmacia que fuesen precisos ${ }^{34}$. En un informe elaborado por el coronel de ingenieros en esta época se comenta la falta de muchas dependencias indispensables, como eran la ropería de entrados, el depósito de armamentos, el cuarto para ropa sucia o el almacén para víveres ${ }^{35}$. No obstante el $1^{\circ}$ de noviembre ya fue aumentado en 600 camas más, lo que daba una dotación de 1.100 camas; y en este mismo mes llegó a las $1.500^{36}$. El 12 de noviembre de 1896 se hizo preciso habilitar como clínica aneja a Regla los almacenes de Santa Catalina. La cuantía del trabajo en el hospital llegó a ser tal que no pudo asumir la visita médica de las guarniciones de esta población, recomendándoles que contrataran los servicios de médicos cubanos civiles ${ }^{37}$.

\footnotetext{
Isla. Finalmente, la dirección, los despachos y los pabellones del director y de los jefes de servicio estaban en otro edificio. La administración militar ocupaba otros 2 más pequeños, y había 4 ó 5 pabellones destinados a jefes, oficiales médicos, ayudantes y personal subalterno. Los sanitarios y enfermeros tenían también 4 ó 5 dormitorios igualmente aislados. Emigrantes chinos, contratados para funciones de limpieza, disponían de su propio departamento en un extremo del hospital. LARRA CEREZO. A. (1898), pp. 23-32.

33 Edificado en la orilla opuesta de la Bahía, estaba en comunicación con la ciudad por medio de vapores que partían cada media hora y con la Isla por la línea férrea de Villanueva. También era punto de llegada de los vapores de evacuación de heridos que recorrían la costa de la provincia de Pinar del Río.

34 Archivo Histórico Militar, Madrid, Sección Ultramar, fondo: Gobiernos Militares, caja 202, Hospitales 1890-1898, Hospital Militar de Regla, 1 legajo.

35 Archivo Histórico Militar, Madrid, Sección Ultramar, fondo: Gobiernos Militares, caja 202, Hospitales 1890-1898, Hospital Militar de Regla, 1 legajo.

36 Ibidem, 2 legajos.

37 Ibidem, 2 legajos.
} 
Del interés real que había en que los servicios se prestaran de una forma adecuada es fiel reflejo una dura carta manuscrita posiblemente por Cesáreo Fernández Losada en noviembre de 1896, dirigida a los directores de los hospitales de Regla y Hacendados, notificándoles que habiéndose recibido noticias de deficiencias en las comidas, subsanasen éstas pues de lo contrario se exigirían responsabilidades ${ }^{38}$. En diciembre de 1896, el director del hospital, Benito Fon, comunica que: «Existiendo un número considerable de enfermos en los colgadizos que dan a la parte sudeste de este hospital, se hace imprescindible y urgente la construcción de retretes en los mismos, donde los enfermos allí existentes puedan satisfacer sus necesidades corporales. Los que ocupan las salas referidas tienen que recorrer un espacio de 150 metros para servirse de los retretes existentes, en los cuales se acumulan gran número de individuos. En muchas ocasiones los enfermos de disentería, catarros intestinales y demás infecciones análogas, no pueden recorrer esa distancia, evacuando sus excrementos sobre el suelo de madera del muelle que ocupan las referidas salas, cuyo espectáculo, además de ser poco moral, crea un foco de infección con las emanaciones que se desprendan de la acumulación de las materias fecales allí depositadas...». Tras esta petición, el cuerpo de ingenieros examinó el hospital y se propuso la construcción de nuevos retretes ${ }^{39}$.

En su peor momento el Hospital de Regla junto a su anexo de Santa Catalina, llegaron a sumar 4896 camas ocupadas; con un promedio de 4500 estancias diarias durante el mes de diciembre de $1896^{40}$. Finalmente debido a la disminución de las enfermedades se trasladaron los enfermos de este hospital a otros, como el de Hacendados, y fue suprimido entre febrero y marzo de 1897. No obstante, a los pocos meses, y de nuevo por el incremento altísimo del número de enfermos, hubo que volver a abrir el Hospital de Regla; de tal modo que en diciembre de 1897 fue preciso, por las necesidades de la campaña, ampliar en 600 camas las 3.000 que ya había alcanzado, contando pues con 3.600 camas. Siendo por tanto el hospital con mayor capacidad de enfermos de toda la Isla ${ }^{41}$.

38 Archivo Histórico Militar, Madrid, Sección Ultramar, Fondo: Gobiernos Militares, Caja 202, 1 legajo.

39 Archivo Histórico Militar, Madrid, Sección Ultramar, Fondo: Gobiernos Militares, Caja 202, Hospitales 1890-1898, Hospital Militar de Regla, 2 legajos.

40 SenAe y Vicente, C. (1898), p. 34.

41 Ibidem, 1 legajo. 


\section{Hospital militar de los Almacenes de Hacendados}

Fue éste uno de los «hospitales provisionales» que se habilitaron en las cercanías de la capital de La Habana para dar cabida a la avalancha de enfermos que se produjo durante 1896.Se creó en unos antiguos almacenes de azúcar, como su nombre indica, el 6 de Noviembre de 1896; recibiendo sus primeros 414 enfermos al día siguiente. Se dotó inicialmente con 1.000 camas, «con objeto de dar expansión a las actuales clínicas que por la aglomeración de enfermos llegados en las últimas 48 horas han dado albergue a mayor número del que pueden contener». Como era de esperar, el hospital fue rápidamente aumentado en otras 1.000 camas, haciendo un total de 2.000 el 25 de noviembre de 1896. Dos días más tarde ya había 1.937 enfermos ingresados. Estos enfermos se distribuían en 10 salas que tenían entre las 100 y las 250 camas cada una, y una sala de cirugía con 127 camas $^{42}$.

Pero esta premura en adecuar sitios a toda costa donde poder instalar a los enfermos daba lugar a serios problemas. Así el 27 de noviembre de 1896 el director de esta institución informa de que debido a la necesidad de dar albergue a todos los enfermos utilizando toda clase de locales cubiertos se tuvieron que instalar 150 camas en la galería exterior de los almacenes que mira al sur, donde los enfermos estaban expuestos a los rayos del sol todo el día, y que como esto era antihigiénico y poco humanitario amenazó con cerrar estas camas a no ser que se le proporcionase un cerramiento adecuado a dicha galería. Apenas diez días antes los responsables del hospital de Hacendados y Regla habían recibido una dura carta desde las instancias superiores sobre las quejas que tenían los enfermos sobre la alimentación. La causa de las deficiencias en la alimentación se debía al hecho de no tener aún realizada la instalación propia de las cocinas, por lo que la comida era enviada desde el Hospital de San Ambrosio, cruzando la bahía de La Habana en barco. El Hospital de Hacendados fue suprimido a los pocos meses de su creación, el 7 de Marzo de 1897, siendo trasladados sus enfermos a otros hospitales, fundamentalmente al Alfonso XIII ${ }^{43}$.

42 Archivo Histórico Militar, Madrid, Sección Ultramar, Fondo: Gobiernos Militares, Caja 202, Hospitales 1890-1898, 1 legajo. Se reproduce en el anexo 2 del capítulo.

43 Todos los datos referentes al Hospital de los Almacenes de Hacendados han sido obtenidos del Archivo Histórico Militar, Madrid, Sección Ultramar, Fondo: Gobiernos Militares, caja 202, Hospitales 1890-1898, Hospital Militar de Hacendados, 14 legajos sin clasificar. 


\section{Hospital Militar de Madera}

Se abrió el Hospital de Madera ${ }^{44}$ en la capital de La Habana el 9 de Agosto de 1896, dotándose inicialmente con 500 camas. Los problemas no tardaron en aparecer. El día 8 de Octubre de 1896 su director Antonio Araoz informó de que los excusados estaban situados contiguamente a la cocina, separados tan sólo por un tabique en mal estado y con agujeros, siendo además los excusados pozos negros malolientes. Entre otros problemas técnicos que fueron solucionados por el Cuerpo de Ingenieros destacan: la construcción de unas garitas para que los soldados de guardia del primer batallón de voluntarios del Cuerpo de Artillería pudieran resguardarse del sol y la lluvia, la reparación de los tejados, la reparación del pararrayos, reparación de los canales de desagüe, retirado de unos cables telefónicos que molestaban al edificio del hospital, etc. De manera que tras el estudio de los problemas referidos, más bien que mal y usando la máxima economía posible, se les iba poniendo remedio.

En los siguientes meses, debido a la continua llegada de enfermos, hubo necesidad de ampliar las instalaciones del mismo. Para dicha ampliación se hizo uso de unas casas cedidas altruistamente por un vecino de la localidad en la calle Marquéz González n ${ }^{\circ} 10$ y 12, dando cabida en las mismas a una clínica de 500 camas para tropa, dependientes del Hospital Militar de Madera, a partir del 13 de noviembre de $1896^{45}$. No obstante los mayores problemas aún estaban por llegar. A medida que avanzaba la guerra los problemas económicos del ejército Español se hacían más acuciantes. El 19 de abril de 1897 el Director daba cuenta de que no había podido suministrar el real para tabaco que se le daba a los enfermos todos los domingos por no haber fondos en la caja del hospital.

En agosto de 1897 el director se dirigió al comisario de la guerra, interventor del hospital, para que le informe de por qué las gallinas que se estaban suministrando a los enfermos no tenían el peso reglamentario, alejándose bastante de los 720 gramos que debían tener. La respuesta fue que no se encontraban mejores en el mercado.

El día 20 de junio de 1898 el director informó que a partir del día siguiente le sería imposible al administrador del hospital procurar el suministro de leche, tanto para las raciones como para la farmacia, por haberse negado los

44 Toda la información referente al Hospital Militar de Madera ha sido obtenida del Archivo Histórico Militar, Madrid, Sección Ultramar, fondo: Gobiernos Militares, caja 202, Hospitales 1890-1898, Hospital Militar de Madera, 35 legajos sin clasificar.

45 Durante esta guerra fue muy frecuente recurrir a aprovechar para instalar ampliaciones de los hospitales o enfermerías militares a cesiones de casas de particulares, y en otras muchas ocasiones al alquiler de éstas. 
proveedores habituales a continuar haciéndolo si no se les pagaba lo que se les adeudaba, y dado además que no había metálico en la caja del hospital para adquirirla al contado y que no se había encontrado a nadie que la quisiera suministrar sin previo pago.

El día 22 de Julio de 1898 cesó el suministro de patatas al hospital por no haber existencias disponibles en La Habana. Como las patatas eran parte importante en la alimentación de los enfermos fue preciso que se reuniera la Junta Facultativa del hospital, la cual determinó sustituir los 173 gramos que corresponden a la ración de patatas por enfermo y día, por tres onzas de arroz.

El día 23 de Julio de 1898 informó el Director que desde el siguiente día no sería posible disponer de las gallinas necesarias para la dieta de los enfermos porque al no haber metálico en la caja el abastecedor se negaba a suministrarlas mientras no se le pagase en efectivo.

El 29 de julio de 1898 el director notifica que: «Habiéndose agotado la existencia de habichuelas, las cuales se suministraban a los enfermos de este Hospital en vez de garbanzos, según tuve la honra de participar a V.E. en mi escrito del día 25 del corriente; he dispuesto que dichas habichuelas sea sustituidas por arroz. En virtud de lo cual desde el día de mañana se compondrá la ración común de: arroz, carne, mitad de tocino y mitad de manteca en cantidad de 43 gramos».

Finalmente los enfermos de este Hospital fueron evacuados al Hospital Alfonso XIII, y se cerró el día 9 de Diciembre de 1898.

\section{Provincia de Matanzas}

La provincia de Matanzas, situada junto a la de La Habana, no dispuso de muchas instalaciones sanitarias debido sobre todo a la proximidad de la ciudad de La Habana a donde eran remitidos con facilidad sus enfermos y heridos. Durante la guerra fueron abiertas las siguientes instalaciones ${ }^{46}$ :

A pesar de estar dotados con este número de camas, el desplazamiento de las operaciones de las columnas militares hacia esta provincia originó el aumento de la morbosidad, llegando a ocuparse el hospital de matanzas con 2127 pacientes y el de Colón con 1878 durante la primavera de $1897^{47}$.

46 La provincia de Matanzas se hallaba comunicada por un ferrocarril central con la capital de la Isla y por el sur enlazaba, igualmente, con ferrocarril con Santa Clara.

47 Senae y Vicente, C. (1898), pp. 40-41. 


\section{HOSPITALES, CLÍNICAS Y ENFERMERÍAS MILITARES DE \\ LA PROVINCIA DE MATANZAS.}

\begin{tabular}{|l|l|l|c|c|}
\hline NomBre & FECHA CREACIÓN & EstABLECIMIENTO & CAMAS & $\begin{array}{c}\text { CAMAS } \\
\text { FINALES }\end{array}$ \\
\hline Colón & 6 Noviembre 1895 & Clínica $\rightarrow$ Hospital & 100 & 600 \\
\hline Matanzas & 21 Enero 1896 & Hospital & 250 & 1.000 \\
\hline Cárdenas & 10 Junio 1896 & Clínica (Matanzas) & 100 & 220 \\
\hline
\end{tabular}

Durante el año 1896 ingresaron 16.907 personas en estos hospitales militares, quedando 891 ingresados al finalizar el $\mathrm{año}^{48}$. A fecha del 1 de Enero de 1898 había una dotación de 1.820 camas en esta provincia, estando ocupadas por enfermos $1.462^{49}$.

\section{Provincia de Santa Clara (Las Villas)}

Esta provincia ocupa la parte central de la isla, siendo una región altamente conflictiva durante esta guerra. Esto motivó la apertura de abundantes hospitales y enfermerías desde el inicio de la contienda ${ }^{50}$. Fue asimismo determinante el avance de las operaciones militares en esta provincia durante el verano de 1897 que repercutió en el aumento de los ingresos hospitalarios.

Durante el año 1896 ingresaron 30.198 enfermos en los hospitales, clínicas y enfermerías militares de esta provincia; permaneciendo ingresados 1.845 al finalizar el año ${ }^{51}$. El número de camas disponibles al iniciarse el año 1898 era de 10.790, sólo la provincia y capital de La Habana le superaba en número, de las cuales permanecían ocupadas 6.049 en ese momento. Por lo que se puede deducir que durante toda la guerra fue una provincia con un alto índice de ocupación hospitalaria ${ }^{52}$.

48 LARra y CEREzo, A. (1901), pp. 18-19.

49 Larra y Cerezo, A. (1898), Appendice I: Carte Sanitaire de l'T̂le de Cuba.

50 Esta zona central de la Isla presentaba más dificultades en su comunicación con el resto de la Isla, y sólo presentaba tramos parciales de línea férrea, como los que unían el puerto de Caibarién con Remedios y Placetas o la que unía Sancti-Spíritus con Tunas de Zaza o Santa Clara con Cienfuegos.

51 LARRA y CEREZO, A. (1901), pp. 17-19.

52 Larra y Cerezo, A. (1898), Appendice I: Carte Sanitaire de l'T̂le de Cuba. 


\section{HOSPITALES, CLÍNICAS Y ENFERMERÍAS MILITARES DE LA PROVINCIA DE SANTA CLARA.}

\begin{tabular}{|l|l|l|c|r|}
\hline NomBRE & FECHA CREACIÓN & ESTABLECIMIENTO & $\begin{array}{c}\text { CAMAS } \\
\text { INICIALES }\end{array}$ & $\begin{array}{c}\text { CAMAS } \\
\text { FINALES }\end{array}$ \\
\hline Santa Clara & Ya existía & Hospital & 300 & 1.000 \\
\hline Placetas & 6 Mayo 1895 & Clínica (Remedios) $\rightarrow$ Hospital & 50 & 900 \\
\hline Sancti-Spíritus & 11 Julio 1895 & Hospital & 150 & 1.500 \\
\hline Remedios & 10 Agosto 1895 & Hospital & 200 & 1.400 \\
\hline Sagua la Grande & 17 Octubre 1895 & Hospital & 150 & 1450 \\
\hline Casilda-Trinidad & 24 Octubre 1895 & Clínica(Sancti-Spíritus) $\rightarrow$ Hospital & 100 & 1.000 \\
\hline Fomento & 26 Enero 1896 & Enfermería $\rightarrow$ Clínica (Placetas) & 50 & \\
\hline Cienfuegos & 28 Abril 1896 & Clínica (Santa Clara) $\rightarrow$ Hospital & 100 & 1.450 \\
\hline Manicaragua & 17 Octubre 1895 & Enfermería $\rightarrow$ Clínica (Santa Clara) & 50 & 100 \\
\hline Cumanayagua & 10 Abril 1897 & Enfermería $\rightarrow$ Clínica (Cienfuegos) & 50 & \\
\hline Yaguajay & 23 Abril 1897 & Clínica (Remedios) & 300 & \\
\hline Tunas de Zaza & 4 Mayo 1897 & Enfermería & 20 & \\
\hline Isabela de Sagua & 4 Mayo 1897 & Hospital & 1020 & \\
\hline
\end{tabular}

\section{Provincia de Puerto Príncipe (CAmagüey)}

La provincia de Puerto Príncipe no dispuso de muchos hospitales, al estar situada entre dos provincias con una alta concentración de establecimientos sanitarios, que eran Santa Clara y Santiago de Cuba. En ella se encontraba la trocha de Júcaro a Morón, tristemente famosa ya en la guerra de 1868 por su alta mortandad, que volvió a ser puesta en uso para impedir el paso de los insurrectos de una parte a otra de la isla ${ }^{53}$.

53 Su nueva puesta en funcionamiento requirió de la presencia de un importante contingente militar lo cual supuso a su vez un aumento de la morbilidad, sobre todo por paludismo, de manera que el Hospital de Ciego de Ávila que ocupaba el centro de la trocha hubo de atender a 6300 soldados durante el mes de noviembre, y 2200 en Morón durante el mismo período, siendo más de la mitad de estos enfermos afectados por el paludismo. SENAE y ViCENTE, C. (1898), pp. 41-42. 


\section{HOSPITALES, CLÍNICAS Y ENFERMERÍAS MILITARES DE LA PROVINCIA de Puerto PRínciPe}

\begin{tabular}{|l|l|l|c|c|}
\hline Nombre & FECHA CREACIÓN & ESTABLECIMIENTO & $\begin{array}{c}\text { CAMAS } \\
\text { INICIALES }\end{array}$ & $\begin{array}{c}\text { CAMAS } \\
\text { FINALES }\end{array}$ \\
\hline Puerto Príncipe & Ya existía & Hospital & 400 & 1.000 \\
\hline Nuevitas & 28 Junio 1895 & Clínica (Puerto Príncipe) & 100 & \\
\hline Guaimaro & 28 Junio 1895 & Clínica (Puerto Príncipe) & 100 & \\
\hline Santa Cruz del Sur & 28 Junio 1895 & Clínica (Ciego de Ávila) & 80 & \\
\hline Ciego de Ávila & 17 Julio 1895 & Hospital & 258 & 1.700 \\
\hline Arroyo Blanco & 19 Julio 1895 & Clínica (Ciego de Ávila) & 100 & 250 \\
\hline Morón & 5 Septiembre 1895 & Clínica $\rightarrow$ Hospital & 25 & 500 \\
\hline Júcaro & 2 Septiembre 1896 & Clínica (Ciego de Ávila) & 150 & \\
\hline Pontón Cortés & 7 Octubre 1895 & Clínica (Puerto Príncipe) & & \\
\hline
\end{tabular}

Durante el año 1896 fueron atendidas 15.671 personas en los hospitales y enfermerías militares de esta provincia. Al finalizar el año permanecían ingresados 813 enfermos $^{54}$. Al iniciarse 1898 los hospitales de esta provincia tenían una capacidad para 3.700 pacientes, siendo el número de camas ocupadas en ese momento $2.605^{55}$.

\section{Provincia de Santiago de Cuba}

Esta provincia era, por delante incluso de La Habana, la que mayor densidad de establecimientos sanitarios presentaba en toda la Isla. Esto era debido a dos motivos, primero al ser el foco inicial de la insurrección y donde ésta se mantuvo con más fuerza durante toda la guerra y segundo a las condiciones climáticas de la zona oriental de la Isla que favorecían el desarrollo de las enfermedades infecciosas, tales como la fiebre amarilla y el paludismo ${ }^{56}$.

54 LARra y CEREZO, A. (1901), pp. 18-19.

55 Larra y Cerezo, A. (1898), Appendice I: Carte Sanitaire de l'Île de Cuba.

56 Otro problema añadido en esta provincia era la dificultad por la orografía de las comunicaciones interiores de esta región, además de existir sólo algún pequeño tramo de ferrocarril, lo cual obligaba a que los establecimientos sanitarios tuvieran una vida más independiente. 


\section{HOSPITALES, CLÍNICAS Y ENFERMERÍAS MILITARES DE LA PRovincia de SANTiago de Cuba.}

\begin{tabular}{|l|l|l|c|c|}
\hline NOMBRE & FECHA CREACIÓN & ESTABLECIMIENTO & $\begin{array}{c}\text { CAMAS } \\
\text { INICIALES }\end{array}$ & $\begin{array}{c}\text { CAMAS } \\
\text { FINALES }\end{array}$ \\
\hline Santiago de Cuba & Ya Existía & Hospital & 400 & 2.000 \\
\hline Báyamo & 24 Abril 1895 & Hospital & 155 & 1000 \\
\hline Holguín & 24 Abril 1895 & Hospital & 155 & 1300 \\
\hline Manzanillo & 24 Abril 1895 & Hospital & 155 & 3000 \\
\hline $\begin{array}{l}\text { Victoria de las } \\
\text { Tunas }\end{array}$ & 19 Mayo 1895 & Hospital & 150 tropa y 5 oficiales \\
\hline Mayarí & 26 Junio 1895 & Hospital & 150 tropa y 5 oficiales \\
\hline Gibara & 28 Junio 1895 & Clínica (Holguín) $\rightarrow$ Hospital & 300 \\
\hline Alto Songo & 29 Julio 1895 & Clínica (Santiago de Cuba) & 30 & 160 \\
\hline San Luis & 29 Julio 1895 & Clínica (Santiago de Cuba) & 30 & 230 \\
\hline Puerto Padre & 9 Agosto 1895 & Hospital & 300 & 500 \\
\hline Guantánamo & 22 Agosto 1895 & Hospital & 150 & 800 \\
\hline Sagua de Tánamo & 3 Septiembre 1895 & Hospital & 100 & 300 \\
\hline Cauto Embarcadero & 14 Septiembre 1895 & Clínica (Manzanillo) & 150 & \\
\hline Jijuaní & 14 Septiembre 1895 & Clínica (Bayamo) & 150 & \\
\hline Veguitas & 14 Septiembre 1895 & Clínica (Manzanillo) & 230 & \\
\hline Firmeza & 3 Diciembre 1895 & Clínica (Santiago de Cuba) & 30 & \\
\hline Baracoa & 5 Diciembre 1895 & Clínica (Santiago de Cuba) & & \\
\hline Palma Soriano & 16 Febrero 1896 & Clínica (Santiago de Cuba) & 150 & \\
\hline San Andrés & 6 Marzo 1896 & Clínica (Holguín) & 50 & \\
\hline Maniabón & 28 Junio 1896 & Hospital & 150 & 200 \\
\hline El Cobre & 7 Julio 1897 & Clínica (Santiago de Cuba) & 100 & \\
\hline Ziguabos & 10 Julio 1897 & Clínica (Guantánamo) & 100 & \\
\hline El Palmar & & & 100 & \\
\hline
\end{tabular}

El hospital militar de Santiago de Cuba era el único que existía previamente al inicio de la guerra, disponía inicialmente de 400 camas, alcanzando en el transcurso de los meses las 2.000. El 24 de Abril de 1895 comenzaron a funcionar instalaciones sanitarias situadas en Báyamo, Holguín y Manzanillo; y el 19 de mayo y 26 de junio respectivamente, en Victoria de las Tunas y Mayarí; 
aunque la apertura oficial de estos cinco establecimientos no se sancionó hasta el 12 de septiembre de 1895, en que por real orden se aprueba la apertura de los mismos $^{57}$. Todos ellos tenían la categoría de hospitales militares, el primero contaba con 300 camas para tropa y 10 para oficiales y el resto con 150 camas para tropa y 5 para oficiales; pero el transcurso de la guerra les fueron aumentando esta dotación hasta llegar a disponer de $1.000,1.300$ y 3.000 camas respectivamente los hospitales de Báyamo, Holguín y Manzanillo.

Manzanillo, Bayamo, Cauto, Veguitas y Guano, fueron hospitales instalados en las proximidades del río Cauto, que por ser navegable, constituía una vía natural de acceso y comunicación con el interior de esta provincia ${ }^{58}$.

Durante el año 1896 fueron atendidos 49.260 enfermos en los hospitales, clínicas y enfermerías militares de esta provincia, al finalizar este año quedaban aún ingresados $3.336^{59}$. Al iniciarse el año 1898 el número de camas de que disponían los establecimientos sanitarios militares de esta parte de la Isla era 10.760 , de las cuales permanecían ocupadas $8.434^{60}$. Debido al alto número de enfermos que existían en esta región, se hizo necesario también habilitar dos antiguos cuarteles de la ciudad de Santiago de Cuba como hospitales. Creándose así en 1897 el hospital del Cuartel de Concha y el hospital Reina Mercedes, dotados de 500 camas cada uno. Asimismo en esta ciudad en el 1898 fue creado el hospital del Barracón, con 300 camas. Siendo todos ellos atendidos por el personal de Sanidad Militar ${ }^{61}$.

Como nota final a estos primeros apartados en que se han comentado los establecimientos sanitarios que hubo en la isla de Cuba, es interesante valorar las reflexiones que un cronista de la época nos ofrece tras su visita en 1896 al Hospital Alfonso XIII de La Habana. Que pueden extrapolarse a la mayoría de los hospitales de la isla que vivieron iguales circunstancias de sobresaturación de enfermos:

«Se hizo para 500, para 800 en caso de apuro y hoy los libros dicen al visitante que allí, aunque hay dos puertas siempre abiertas, la de entrada y la de salida, existen 2.867 entre enfermos y heridos.

57 «Real Orden Circular 12 septiembre 1895», Diario Oficial del Ministerio de la Guerra, Madrid, Tomo I, 1895,

58 SenAe y ViCENTE, C. (1898), pp. 50-51.

59 LARRA y CEREZO, A. (1901), pp. 18-19.

60 Larra y Cerezo, A. (1898), Appendice I: Carte Sanitaire de l'Île de Cuba.

61 GuERrA, F. (1994), pp. 156, 159. 
Todavía son pocos, aún pueden amontonarse más mientras la dirección sanitaria busca otros locales, un local cualquiera, porque ya no hay que reparar en nada para buscar albergue (...)

(...) Lo que está a mi alcance, lo que veo sin que nadie me lo indique, lo que procuro anotar sin que nadie me vea, confundido entre aquellos soldados que van ocupando la sala, revuelto entre sanitarios y médicos, entre enfermeros y hermanas de la Caridad, es que son muchos 3000 enfermos para un hospital de 500, de 800 quizás en caso de apuro.

Veo que un médico tiene a su cuidado 150, hasta 200 alguno; que la botica ha de despachar 3.000 recetas diarias y esto si no se da a cada enfermo un sólo medicamento; la cocina ha de despachar otras 3.000 raciones si es que cada enfermo ha de comer una sola vez al día; que son pocos los médicos y mucho el trabajo, y que si no hay conflictos graves es porque todos se sacrifican por el enfermo.

(...) Dejo la ciudad de madera, tan bonita, tan alegre, tan sana, con todos sus horrores de la aglomeración y su personal sacrificado, lo dejo con pena viendo como va inutilizándose una casa que era admirable» ${ }^{62}$.

A lo largo de la campaña se intentó mejorar en lo posible la situación sanitaria de las tropas, tal y como puede apreciarse en esta real orden cursada por el Ministro de la Guerra:

«Excmo. Sr.: En vista del telegrama de V.E. del 4 del actual, referente al estado sanitario del ejército de esa Isla, y a fin de mejorarlo en cuanto sea posible, el Rey (Q.D.G.), y en su nombre la Reina Regente del Reino, se ha servido autorizar a V.E. para que, informado con urgencia el Cuerpo de Sanidad militar y las autoridades que juzgue competentes, disponga cuanto sea necesario:

$1^{\circ}$. Para mejorar el acuartelamiento, alimentación y vestuario de las tropas.

$2^{\circ}$. Aplicar todas las medidas higiénicas convenientes y compatibles con las necesidades de las operaciones de campaña que aconseje el Cuerpo de Sanidad Militar y aprueba V.E., llamando su atención sobre el uso de filtros para las aguas y aireación de éstas para oxigenarlas.

$3^{\circ}$. Establecer sanatorios para convalecientes y débiles o enfermizos en la Isla de Pinos, Guanabacoa, Santa María del Rosario en La Habana, Nuevitas en Puerto Príncipe, y el Cobre en Cuba, como también en otros puntos de la Isla en que, oído el Cuerpo de Sanidad militar, y dada la situación de las tropas, lo considere conveniente.

$4^{\circ}$. Instalar nuevos hospitales, ampliar los actuales y reducir los que hoy excedan de 1.000 enfermos, distribuyendo el excedente de este número en otros hospitales existentes o de nueva creación.

$5^{\circ}$. Crear clínicas dependientes de hospitales en los puntos de fácil comunicación marítima, fluvial o por vía férrea con estos mismos hospitales.

62 Estos párrafos están extraídos de: GuERrero, R. (1896), Crónicas de la guerra de Cuba, Barcelona, Librería Editorial de M. Maucci, 5 vols., Tomo IV, pp. 335-338, 
$6^{\circ}$. Establecer enfermerías regimentarias de 30 a 50 camas, siendo el material y utensilio de instalación, así el suministrado por Administración militar como el clínico, quirúrgico y utensilio de farmacia, facilitado por Sanidad militar, con cargo al crédito extraordinario de campaña.

$7^{\circ}$. Disponer que las clases e individuos de tropa convalecientes y enfermizos, cuyo estado no exija la repatriación, pasen a los sanatorios hasta reponer sus fuerzas; que los enfermos leves y afectos de dolencias de corta duración y los cansados inaptos por el momento para el servicio activo y que con un breve descanso han de recuperar sus fuerzas, sean asistidos en las enfermerías regimentarias y en las clínicas; que los enfermos que deban causar mayor número de estancias, pasen a los hospitales; que la evacuación de estos se haga con la oportunidad necesaria para que en ningún tiempo exceda el número de enfermos de la capacidad de los locales disponibles; que se evite siempre la acumulación de más de 1.000 enfermos en un solo hospital, y últimamente que se active la tramitación de las propuestas de inútiles y pase a la Península, formulándose aquellas apenas comprobado el diagnóstico y juzgada necesaria la repatriación, aplicando los reglamentos de exenciones y las Reales Órdenes vigentes de pase por enfermo a la Península, con criterio amplio por lo que se refiere a los tuberculosos y predispuestos a esta misma enfermedad, a los afectos de debilidad y demacración general consecutiva a graves enfermedades, a los palúdicos con lesiones hepáticoesplénicas, a los anémicos ya graduados y a los dispépsicos, diarreicos y disentéricos confirmados ${ }^{63}$.

\section{CONCLUSIONES}

Este recorrido por los establecimientos sanitarios que tuvo el Cuerpo de Sanidad Militar en Cuba durante la guerra, nos da una idea del esfuerzo desarrollado para atender al elevado número de enfermos del ejército español. Se actuó con versatilidad adaptándose a las situaciones cambiantes de la guerra, abriéndose o ampliándose clínicas, hospitales y enfermerías según las necesidades de la campaña ${ }^{64}$. A lo largo de este trabajo la impresión general es que se intentó hacer todo lo técnica y humanamente posible por atender adecuadamen-

$63 \ll$ Real Orden 6 Noviembre 1897»Diario Oficial del Ministerio de la Guerra, № 251, tomo IV, pp. 940-

64 El sistema sanitario fue fruto de las necesidades que la campaña fue creando y también mediatizado por las comunicaciones, férreas y marítimas sobre todo, y por la orografía del país; ya que fue siempre fundamental el papel dado a poder favorecer la evacuación y recepción final de enfermos con dirección a La Habana, último centro receptor y único punto desde el que podían ser repatriados los soldados a España. 
te al soldado español, pero la avalancha de enfermos superó con creces cualquier cálculo remotamente imaginable, por lo que hubo también que improvisar sobre la marcha habilitando como clínicas u hospitales en locales poco adecuados para esa finalidad, clínicas que hubieron de ampliar al máximo su capacidad, sacando espacio y camas donde no las había y los profesionales duplicando su trabajo bajo una demanda asistencial muy por encima de lo normal ${ }^{65}$.

65 Felipe Ovilo Canales, médico militar presente en aquella campaña escribía a este respecto: «La organización sanitaria de un ejército -como he dicho repetidas veces, y he de repetir más, no puede improvisarse... Fueron aquellos días de verdadera angustia, tanto para el que mandaba como para los que obedecían; en ellos se excedieron todos para remediar en lo posible aquel desastre, más dañino que una derrota en los campos de batalla; la aglomeración de enfermos en número infinitamente superior a todas las previsiones, originó muchas faltas, muchísimas; pero ciertamente menores a las que hubieran podido esperarse, gracias a los esfuerzos de todos». Ovilo CANales, F. (1899), La decadencia del Ejército. Estudio de Higiene Militar, Madrid, Imprenta y Litografía del Hospicio, pp. 29-30. 